\title{
COMUNICACIÓN
}

\section{El problema de la pediculosis capitis en escolares del área metropolitana de San José, Costa Rica}

\author{
OLGER CALDERÓN-ARGUEDAS*, MAYRA E. SOLANO* y CLAUDIO SÁNCHEZ** \\ THE PROBLEM OF PEDICULOSIS CAPITIS IN SCHOOLCHILDREN OF THE \\ METROPOLITAN AREA OF SAN JOSE, COSTA RICA.
}

The problem of pediculosis capitis was studied in 28 schools of the metropolitan area of San Jose, Costa Rica. 7.312 children were evaluated by naked eye observation (NEO) and a sample of hair or presuntive parasite forms was collected in all the suspected cases of pediculosis capitis. This material was cleared and mounted in Hoyer's medium for microscopic observation. 730 (10.0\%) of the tested children were positive at least for one of the following parasite forms: hatched or non-hatched eggs, nymphs and adults. 590 (80.8\%) of the positive children were girls and 140 (19.2\%) were boys.The prevalence rates observed in the studied schools showed values between 3.2 and 27.2\%. These data suggest that pediculosis capitis is one of the most common public health problems in our children, that can interfere with their learning processes and general welfare.

Key words: Pediculus humanus capitis, pediculosis capitis, ectoparásitos, Costa Rica.

\section{INTRODUCCIÓN}

La pediculosis capitis es una ectoparasitosis producida por Pediculus humanus capitis (Anoplura: Pediculidae), el cual es un insecto hematófago altamente estenoxénico ${ }^{1}$. El cuero cabelludo humano le provee de un ambiente controlado con una temperatura de aproximadamente $30^{\circ} \mathrm{C}$ y una humedad relativa del $70 \%$, fuera del cual ni los huevos ni las formas vegetativas de parásito pueden sobrevivir ${ }^{1}$. Es aquí donde realizan sus ciclos de alimentación por medio de picadas que se repiten aproximadamente cada cuatro horas, generando un intenso prurito en los hospedadores. Las escoriaciones dérmicas como consecuencia del rascado usualmente se suelen infectar de forma secundaria ${ }^{1}$.

La pediculosis es un problema de salud pública cosmopolita que ha afectado a la humanidad a largo de toda su historia ${ }^{2,3}$. Su alta prevalencia ha sido relacionada con la limitada disposición de agua y deficientes prácticas de aseo personal ${ }^{4}$. En la cuarta y quinta década del siglo XX, el mejoramiento global en los suministros de agua potable, las prácticas cada vez más frecuentes de baño corporal, el lavado de cabeza, el empleo de ropa de cama para dormir y el uso de pelo corto en los varones minimizó significativamente la situación ${ }^{4}$.

A partir de los años 60 se observó un marcado aumento en la prevalencia mundial de la parasitosis, que algunos la relacionan con las

\footnotetext{
* Centro de Investigación en enfermedades tropicales (CIET). Departamento de Parasitología, Facultad de Microbiología, Universidad de Costa Rica.

** Hospital de Los Chiles, Alajuela, Costa Rica. Caja Costarricense de Seguro Social
} 
nuevas características en los modos de vida de ciertas subculturas que se dieron a partir de ese momento. Dentro de estos figuran la declinación en los hábitos de higiene personal, el incremento en la promiscuidad y el uso del cabello largo principalmente en la población joven ${ }^{4}$. Aunque la infestación se ha relacionado con las clases bajas, también se ha podido evidenciar en personas pertenecientes a las otras clases sociales ${ }^{1}$.

En Costa Rica, las políticas de salud implementadas a partir de los años setenta tuvieron un impacto significativo en la disminución de la transmisión de ciertas enfermedades infecto contagiosas como las parasitosis intestinales, sin embargo la pediculosis sigue siendo un problema que se aprecia con bastante frecuencia en la población costarricense.

Dado que no existen datos que muestren la magnitud del problema en Costa Rica, se decidió implementar el presente estudio en una muestra de escolares, los cuales constituyen el grupo etáreo más afectado por esta parasitosis ${ }^{3}$.

\section{MATERIAL Y MÉTODOS}

Se estudió la ocurrencia de infestación por $P$. humanus capitis durante de los ciclos lectivos de 1995 a 1997 en 28 escuelas públicas diurnas del Gran Area Metropolitana de San José, Costa Rica, las cuales fueron seleccionadas de forma aleatoria. En cada uno de los centros educativos se seleccionaron entre el 35 al $45 \%$ de los niños que conformaron la población estudiantil, los cuales fueron sometidos al proceso de estudio. La presencia de infestación fue determinada mediante observación al ojo desnudo (NEO) según método previamente descrito ${ }^{5}$. A cada niño presuntivamente positivo, se le tomó una muestra de cabello o de las formas parasitarias observadas. Este material fue aclarado en lactofenol claro y montado en Medio Hoyer para su posterior observación microscópica.

Los porcentajes de positividad entre niños y niñas fueron comparados mediante una prueba de hipótesis para comparación de proporciones utilizando un coeficiente de confiabilidad del $95 \%{ }^{6}$.

\section{RESULTADOS}

La infestación por $P$. humanus capitis fue evidenciada en 730 de 7.312 niños evaluados lo que demuestra una prevalencia global del 10,0\%
(Tabla 1). La totalidad de los centros educativos estudiados fue positiva por la infestación y las tasas de prevalencia fluctuaron entre el 3,2 y el $32,4 \%$ (Tabla 1).

Del total de positivos, $590(80,2 \%)$ fueron niñas y $140(19,2 \%)$ fueron niños (Figura 1). Los porcentajes de positividad mostraron diferencias estadísticamente significativas entre ambos sexos $(\mathrm{p}<0,05)$.

\section{DISCUSIÓN}

En el presente estudio se demuestra que la infestación con $P$. humanus capitis es frecuente en las escuelas públicas del casquete urbano de San José. (Tabla 1). A pesar de que en muchos centros educativos la prevalencia fue baja, cabe destacar que en tres de los mismas, esta fue superior al $25 \%$ (Tabla 1). La prevalencia global fue del $10,0 \%$.

Durante la década de los 90, se han efectuado varios estudios sobre pediculosis en escolares y preescolares. En uno de ellos, se encontró un $16,0 \%$ de prevalencia en 10.562 infantes provenientes de jardines de niños, escuelas primarias y colegios secundarios básicos del condado de Youlin, Taiwan ${ }^{5}$. Otro estudio realizado en escolares aborígenes del norte de Taiwan ${ }^{8}$ mostró una tasa de prevalencia del $12,8 \%$ en 2.725 niños procedentes de 35 escuelas primarias pertenecientes a diferentes condados. En un trabajo realizado en 346 niños de 4 escuelas primarias de Kangwon-do, Korea, se encontró na prevalencia global de 37,2 \%9. Las anteriores referencias, al igual que el presente estudio demuestran que el problema de la pediculosis en niños sigue siendo frecuente en diversos lugares del mundo.

Los datos relativos a sexo observados en el presente estudio (Figura 1) sugieren que el patrón de infestación es el mismo que se ha observado en otras investigaciones, en las cuales la mayoría de la población afectada está representada por niñas ${ }^{1,9-11}$. La preferencia por el sexo femenino se ha relacionado con las características de longitud del cabello que suelen portar las niñas, en donde los cabellos largos facilitan la transmisión de las formas infestantes de un hospedador a otro.

A pesar de que la pediculosis puede ser asintomática, en la mayoría de los casos la ocurrencia de la infestación se relaciona con un cuadro de prurito intenso, fundamentalmente en 
Tabla 1. Prevalencia de infestación por Pediculus humanus capitis en los niños de los centros educativos estudiados

\begin{tabular}{|c|c|c|c|c|c|}
\hline & $\begin{array}{l}\text { Centro } \\
\text { educativo }\end{array}$ & $\begin{array}{c}\text { Año de } \\
\text { muestreo }\end{array}$ & $\begin{array}{c}\mathbf{N}^{\circ} \text { de estudiantes } \\
\text { encuestados }\end{array}$ & $\begin{array}{c}\mathrm{N}^{\circ} \text { de estudiantes } \\
\text { con } \\
\text { infestación positiva }\end{array}$ & $\begin{array}{r}\text { Tasa de } \\
\text { prevalencia } \\
\text { en \% }\end{array}$ \\
\hline 1 & MF & 1996 & 314 & 10 & 3,2 \\
\hline 2 & $\mathrm{RV}$ & 1996 & 318 & 31 & 9,7 \\
\hline 3 & $\mathrm{BC}$ & 1995 & 385 & 32 & 8,3 \\
\hline 4 & $\mathrm{JM}$ & 1995 & 373 & 18 & 4,8 \\
\hline 5 & $\mathrm{MH}$ & 1997 & 411 & 23 & 5,6 \\
\hline 6 & B & 1997 & 77 & 25 & 32,4 \\
\hline 7 & VM & 1995 & 279 & 21 & 7,5 \\
\hline 8 & $\mathrm{RC}$ & 1997 & 382 & 19 & 5,0 \\
\hline 9 & CL & 1997 & 287 & 49 & 17,0 \\
\hline 10 & $\mathrm{AL}$ & 1997 & 220 & 42 & 19,1 \\
\hline 11 & SF & 1997 & 286 & 51 & 17,9 \\
\hline 12 & $\mathrm{JJ}$ & 1996 & 150 & 22 & 14,6 \\
\hline 13 & DS & 1997 & 293 & 46 & 15,7 \\
\hline 14 & $\mathrm{C}$ & 1996 & 133 & 16 & 12 \\
\hline 15 & IC & 1997 & 219 & 25 & 11,4 \\
\hline 16 & RH & 1996 & 445 & 54 & 12,1 \\
\hline 17 & RV & 1997 & 104 & 5 & 4,8 \\
\hline 18 & UPJA & 1997 & 307 & 50 & 16,3 \\
\hline 19 & MAL & 1997 & 152 & 15 & 9,9 \\
\hline 20 & JAVR & 1997 & 46 & 12 & 26,1 \\
\hline 21 & LP & 1996 & 162 & 25 & 15,4 \\
\hline 22 & $\mathrm{BH}$ & 1996 & 76 & 21 & 27,6 \\
\hline 23 & $\mathrm{AB}$ & 1997 & 324 & 30 & 9,2 \\
\hline 24 & $\mathrm{AC}$ & 1996 & 313 & 10 & 3,2 \\
\hline 25 & CS & 1997 & 478 & 35 & 7,3 \\
\hline 26 & NU & 1997 & 226 & 16 & 7,1 \\
\hline 27 & NQ & 1997 & 402 & 21 & 5,2 \\
\hline 28 & $\mathrm{CR}$ & 1996 & 150 & 6 & 4,0 \\
\hline Total & & & 7312 & 730 & 10.0 \\
\hline
\end{tabular}

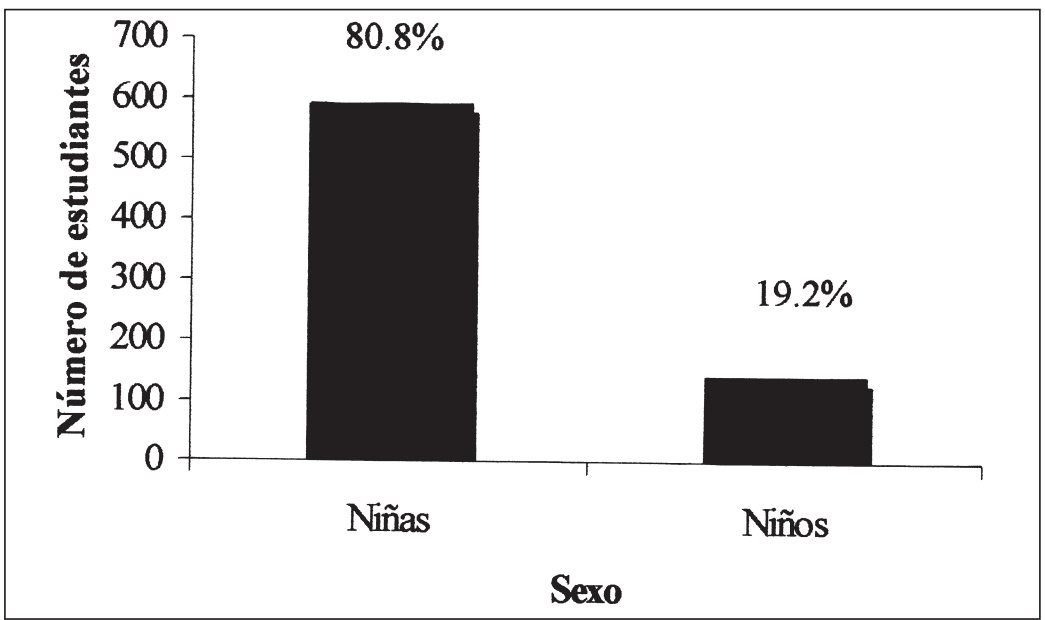

Figura 1. Número de escolares positivos por P. humanus capitis de acuerdo al sexo que se observaron en la muestra de estudio. Los porcentajes de positividad mostraron diferencias estadísticamente significativas entre ambos sexos $(\mathrm{p}<0,05)$. 
la porción retroauricular y occipital de la cabeza, acompañado de lesiones por rascado que muy a menudo se infectan con piógenos. Estas situaciones son una causa de estrés y estigmatización social que puede interferir con los procesos de aprendizaje y a la vez minar el bienestar general de la población infantil.

$\mathrm{Al}$ igual que lo han concluido otros estudios, aparte del tratamiento adecuado con fármacos, se debe hacer un particular énfasis en la prevención y en la información, fundamentalmente a los padres, para poder minimizar efectivamente la ocurrencia de esta parasitosis.

\section{RESUMEN}

El problema de la pediculosis capitis fue estudiado en 7.312 niños procedentes de 28 escuelas del Area Metropolitana de San José, Costa Rica., mediante observación al ojo desnudo (NEO). De los casos presuntivos se tomó un muestra de cabello o de los supuestos parásitos. Este material fue aclarado y montado en medio Hoyer para su posterior observación microscópica. Se encontraron, $730(10,0 \%)$ niños positivos al menos por una de las siguientes formas parasitarias: huevos eclosionados o no eclosionados, ninfas y adultos de $P$. humanus capitis. $590(80,8 \%)$ de los positivos fueron niñas y $140(19,2 \%)$ fueron niños. Las tasas de prevalencia observadas en las escuelas estudiadas mostraron valores entre el 3,2 y el $27,2 \%$.

Los datos obtenidos sugieren que la pediculosis capitis es uno de los problemas de salud pública más comunes en nuestros niños el cual puede interferir con los procesos de aprendizaje y el bienestar general de la población infantil.

\section{REFERENCIAS}

1.- DOWNS A, STAFFORD K, COLE G. Head lice: Prevalence in schoolchildren and insecticide resistance. Parasitol Today 1999; 15: 1-4.
2.- SCHENONE H, LOBOS M. Pediculosis capitis, un permanente y renovado problema. Bol Chile Parasitol 1997; 52: 73-6.

3.- MUMCUOGLU K. Prevention and treatment of head lice in children. Ped Drugs 1999; 1: 211-8.

4.- SCHENONE H, SAAVEDRA T, ROJAS A. Infestación por Pediculus humanus capitis. Un prolongado problema de salud pública. Bol Chile Parasitol1986; 41: $16-20$.

5.- FANG P, CHUNG W, KUO C, et al . Present status of head louse (Pediculus capitis) infestation among schoolchildren in Yunlin County, Taiwan. Kaohsiung J Med Sci 1991; 7: 151-9.

6.- DANIEL W. Pruebas de hipótesis. En: Bioestadística. Base para el análisis de las ciencias de la salud. Editorial Limusa, México, 1988. 667 pp.

7.- DANIEL W. La distribución ji cudrada y el análisis de frecuencias. En: Bioestadística. Base para el análisis de las ciencias de la salud. Editorial Limusa, México, 1988. $667 \mathrm{pp}$.

8.- FANG P, CHUNG W, FANG C et al. Prevalence and treatment of Pediculus capitis infestation among aboriginal school children in northern Taiwan. Kaohsiung J Med Sci 1999; 15: 209-17.

9.- HUH S, PAI K, LEE S et al. Prevalence of head louse infestation in primary school children in Kangwondo, Korea. Korean J Parasitol 1993; 31: 67-69.

10.- HONG H, KIM C, LEE J et al. Infestation rate of head lice in primary school children in Inchon, Korea. Korean J Parasitol 1995; 33: 243-4.

11.- HUH S, KOOK J, CHAI J, PAI K. Infestation rate of lice in patients in a mental hospital, Seoul, Korea. Korean J Parasitol 1994; 32: 275-6.

12.- HARDWOOD R, JAMES M. The lice. En: Entomology in human and animal health. $7^{\text {th }}$ edition. The Macmillan Publishing Co, Inc. New York, 1979. 548 pp.

Agradecimientos: Los autores quieren expresar su agradecimiento a las estudiantes Hazel Rojas Gómez y Jacqueline Moya Chinchilla, por su labor de compilación y organización de los datos concernientes a esta investigación, a los estudiantes de la Universidad de Costa Rica que participaron en el Trabajo Comunal Universitario TCU$05310 \mathrm{~B}$ «La pediculosis y otras ectoparasitosis en el Area Metropolitana de San José», a la sección de Trabajo Comunal Universitario de la Vicerrectoría de Acción Social de la Universidad de Costa Rica por el apoyo logístico a dicho proyecto y al Ministerio de Educación Pública de Costa Rica por permitir la ejecución de esta investigación en los centros educativos seleccionado. 